\title{
Dual Connectivity for LTE-NR Cellular Networks: Challenges and Open Issues
}

\author{
Roberto P. Antonioli, Gabriela C. Parente, Carlos F. M. e Silva, Diego A. Sousa, \\ Emanuel B. Rodrigues, Tarcisio F. Maciel, Fco. Rodrigo P. Cavalcanti
}

\begin{abstract}
The Dual Connectivity (DC) technology has gained a lot of momentum in the LTE Release 12 as a means to enhance the per-user throughput and provide mobility robustness. Some studies in the literature have discussed the interworking between the LTE and the air interface of the upcoming Fifth Generation (5G) in a DC scenario. That integration may provide some benefits to meet the high throughput, reliability and availability requirements of the $5 \mathrm{G}$ networks. This work firstly presents a brief overview of the DC technology considering the integration between 4G e 5G. Then, we highlight some open issues and challenges for future investigation involving Radio Resource Management (RRM) in such a scenario, which includes: (i) user-cell association, (ii) interaction between base stations and (iii) resource allocation. After that, we propose an extension of a utility-based resource allocation algorithm for DC scenarios. Finally, a performance evaluation is conducted in multi-Radio Access Technology (RAT) LTE-NR scenarios considering the bearer split configuration of the DC technology, where the gains provided by the proposed algorithm are demonstrated.
\end{abstract}

Index Terms-5G, dual connectivity, heterogeneous networks, multiple radio access technologies, radio resource management.

\section{INTRODUCTION}

Due to the steep increase in mobile traffic over the past years, there have been many attempts to find new communication technologies that further improve the end-user experience and system performance of mobile networks. The traffic growth has been mainly driven by the explosion in the number of connected devices, which are always demanding more high-quality content that requires very high transmission rates. This resulted in a 4000-fold growth in mobile traffic over the past 10 years [2]. As a consequence, industry and academy have triggered investigations to develop new technologies to meet the forecasted capacity demands.

One of the most promising alternatives to achieve the ultra-high per-user throughput demands is to increase the cell densification by deploying small cells (known as pico cells and femto cells) [3], which have smaller coverage region and

This work was supported by Ericsson Research, Wireless Access Network Department, Sweden and Ericsson Innovation Center, Brazil, under UFC.43 Technical Cooperation Contract Ericsson/UFC. The authors would like to thank CAPES, CNPq, and FUNCAP for their financial and scholarship support.

The authors are with the Wireless Telecommunications Research Group (GTEL), Federal University of Ceará (UFC), Fortaleza, Ceará, Brazil.

Diego A. Sousa is also with Federal Institute of Education, Science, and Technology of Ceará, Paracuru, Ceará, Brazil.

E-mails: \{antonioli, gabriela, cfms, diego, emanuel, maciel, rodrigo\}@gtel.ufc.br.

A preliminary version of this paper was presented in XXXV Simpósio Brasileiro de Telecomunicações (SBrT'17), São Pedro, SP, Brazil, Sep. 2017 [1].

Digital Object Identifier: 10.14209/jcis.2018.28

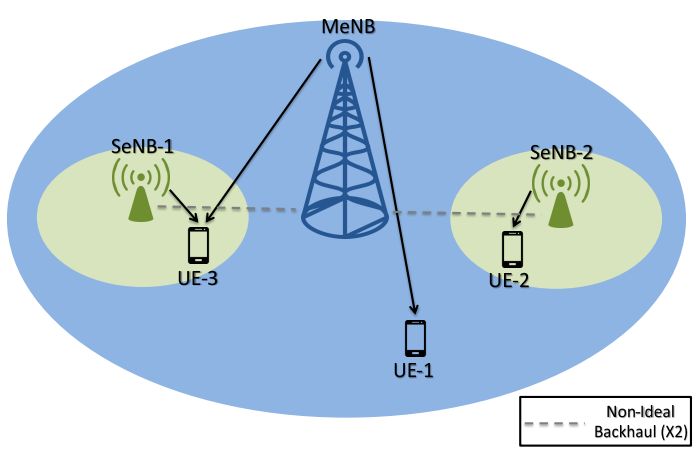

Fig. 1. Example of deployment scenario of DC composed of one MeNB and two SeNBs. Notice that the UE-1 and UE-2 are in Single Connectivity (SC) with the MeNB and SeNB-2, respectively, while UE-3 is in DC with the SeNB-1 and MeNB.

lower transmit power if compared to traditional macro cells (deployments and requirements for small cells can be found in [4]). In these Heterogeneous Networks (HetNets), the macro cells are responsible for providing a wide and reliable coverage region, while the small cells can offer improved capacity in hotspot areas and offload some traffic from the macro cell [5]. However, the deployment of small cells has the disadvantage that, due to the smaller cell coverage area and the larger number of cell boundaries, mobility-related issues may arise, such as an increase in the number of cell (re)selections and handovers.

In this context, the Dual Connectivity (DC) technology has been proposed in the Long Term Evolution (LTE) Release 12 specifications by 3rd Generation Partnership Project (3GPP) as one of the most relevant technologies to accomplish even higher per-user throughput and mobility robustness, and load balancing [6]. Given that a User Equipment (UE) is configured with DC, it can be connected simultaneously to two Evolved Node Bs (eNBs): a Master eNB (MeNB) and a Secondary eNB (SeNB), which operate on different carrier frequencies and are interconnected by traditional backhaul links (known as $\mathrm{X} 2^{1}$ interface in accordance with the LTE terminology). These X2-based backhauls are non-ideal in practice, being characterized by a certain latency and limited capacity [4].

In Fig. 1, an example of a DC scenario is illustrated, which is composed of a MeNB connected to two SeNBs via non-ideal backhaul links and three UEs. UE-1 and UE-2 are in Single Connectivity (SC) with MeNB and SeNB-2,

\footnotetext{
${ }^{1}$ The X2 interface connects two neighboring eNBs in a peer to peer fashion to assist handover and provide a means for rapid co-ordination of radio resources.
} 
respectively, while UE-3 is in DC with the SeNB-1 and MeNB. Therefore, the throughput of UE-3 would be increased by utilizing radio resources from different eNBs.

Differently from the DC scenario presented in [3] and [6], where a HetNet composed of LTE eNBs operating on different frequencies was considered, another possible solution for DC that has been exploited in the literature is a scenario with the integration between multiple Radio Access Technologies (RATs), where the MeNB belongs to one RAT and the SeNB to another. In this context, some works have considered as a possible solution for DC a tight integration between the upcoming Fifth Generation (5G) RAT, named New Radio (NR), and the legacy Fourth Generation (4G) RAT, namely LTE [7], [8].

More specifically, in the LTE-NR integration, the legacy LTE MeNB would provide a larger and more reliable coverage region to the SeNBs using the NR technology [8], [9]. Therefore, for simplicity, in the remaining of this work, MeNB refers to LTE MeNB and SeNB refers to NR SeNB. The objective of this configuration is to increase the system reliability by diminishing the occurrence of service interruptions that might occur due to the intrinsic propagation characteristics of millimeter Wave $(\mathrm{mmW})$ used by $\mathrm{NR}$ (such as higher penetration loss, lower diffraction, and signal blocking from moving objects) or because of Non-Line of Sight (NLOS) situations when using narrow beams with massive Multiple Input Multiple Output (MIMO). Besides, this integration targets the fulfillment of the $5 \mathrm{G}$ requirements by means of allowing simultaneous multi-RAT connectivity in order to provide faster mobility and Centralized/Common Radio Resource Management (CRRM) [9].

The proposed tight interworking between the LTE and NR technologies goes beyond the current inter-RATs cooperation, where slow procedures allow hard handover and access selection procedures, and are focused on coverage purposes [8]. Furthermore, the LTE-NR tight integration would enable the exploration of: (i) RAT diversity, where either the best RAT or simultaneously multiple RATs would be selected for establishing connection, and (ii) transmission diversity, where the same packet would be transmitted via both RATs to enhance reliability or different packets would be transmitted via the different RATs to increase the per-user throughput.

Considering this multi-RAT and multi-connectivity scenario, the contributions of the present work are as follows:

1) This work firstly presents a brief overview of the DC technology based on the concepts presented in [6] and considering the integration between LTE and NR [1].

2) Furthermore, some challenges involving Radio Resource Management (RRM) techniques in a DC scenario are discussed. In this discussion, we present a comprehensive survey involving each step of the RRM for DC scenarios, highlight the drawbacks of the works found in the literature, and discuss challenges and open issues related to RRM algorithms. We also describe how the use cases envisioned to be present in $5 \mathrm{G}$ scenarios can benefit from the DC technology.

3) We also propose an extension of a utility-based resource allocation algorithm for DC scenarios. To perform this extension, we consider an assumption approved in 3GPP discussions and already used in the literature.

4) Finally, we conduct a performance evaluation in multi-RAT scenarios based on the bearer split configuration of the DC technology and composed of LTE-NR eNBs, where the gains provided by the proposed algorithm are demonstrated. To the best of our knowledge, this is the first work in the literature conducting a performance evaluation in such scenario.

The rest of this work is organized as follows. In Section II, we present the 5G use cases and some Key Performance Indicators (KPIs) for $5 \mathrm{G}$ networks. In Section III, an overview of the DC technology is presented following the 3GPP recommendations of Release 12 and recent works from the literature. In Section IV, the user connectivity solutions are presented considering the new DC scenario. Challenges involving RRM techniques in the LTE-NR scenario with DC are presented in Section V. Section VI presents the performance evaluation considering $5 \mathrm{G}$ multi-RAT scenarios. Finally, in Section VII, the conclusions and perspectives of future work are drawn.

\section{Use CASES AND KPIS FOR 5G NeTwORKS}

5G networks are expected to support a wide range of applications/services, which poses new challenges on the current network technologies and in terms of RRM to meet the diversified demands. The applications expected for the $5 \mathrm{G}$ era have been categorized in three different use cases by the International Telecommunication Union (ITU) [10]:

- Enhanced Mobile Broadband (eMBB) - which includes services that will require seamless multi-connectivity across different RATs operating over a wide range of frequency bands (from sub-6GHz to $\mathrm{mmW}$ ), and require very high throughputs and large bandwidths, such as $4 \mathrm{~K}$ and $3 \mathrm{D}$ video, virtual and augmented reality, etc. Therefore, this category is focused on meeting people's demand for an ever increasing digital lifestyle;

- Ultra-Reliable and Low-Latency Communications (URLLC) - that comprises vehicular communication (e.g., to support autonomous cars) and remote control (e.g., remote robotics, medical surgery or tactile Internet), which are applications related to the digital industry and demand very low latency, and very high reliability and availability;

- Massive Machine-Type Communications (mMTC) that involves applications for a further developed digital society with a large number of connected devices transmitting small amounts of data such as in smart cities and smart home/building, which are characterized by requiring low bandwidth, high connection density, enhanced coverage, and low energy consumption.

In order to support the rigorous requirements of the upcoming eMBB, URLLC and mMTC applications, the ITU and 3GPP have been discussing more strict values for some KPIs [10], [11]. Some of the most important KPIs are shown in Table I.

The services/applications from the eMBB and URLLC use cases may benefit from the DC technology in order to fulfill the requirements presented in Table I and the other requirements 
TABLE I

KPIS VALUES EXPECTED FOR 5G NETWORKS.

\begin{tabular}{llll}
\hline KPI & Use case & Target value & Ref.'s \\
\hline \multirow{2}{*}{ Peak data rate ${ }^{\mathrm{a}}$} & eMBB & $10 \mathrm{Gbps}$ for UL & {$[10],[11]$} \\
& eMBB & $20 \mathrm{Gbps}$ for DL & {$[10],[11]$} \\
\hline \multirow{3}{*}{ User plane latency } & eMBB & $4 \mathrm{~ms}$ for UL and DL & {$[10],[11]$} \\
& URLLC & $0.5 \mathrm{~ms}$ for UL and DL & {$[11]$} \\
\hline \multirow{2}{*}{ User experienced data rate } & URLLC & $1 \mathrm{~ms}$ for UL and DL & {$[10]$} \\
\hline \multirow{3}{*}{ Mobility } & eMBB (dense urban) & $50 \mathrm{Mbps}$ for UL & {$[10]$} \\
& eMBB (dense urban) & $100 \mathrm{Mbps}$ for DL & {$[10]$} \\
\hline Mobility interruption time & High speed vehicular & $120 \mathrm{~km} / \mathrm{h}$ to $500 \mathrm{~km} / \mathrm{h}$ & {$[10],[11]$} \\
& Vehicular & $10 \mathrm{~km} / \mathrm{h}$ to $120 \mathrm{~km} / \mathrm{h}$ & {$[10]$} \\
\hline Battery life & Pedestrian & $0 \mathrm{~km} / \mathrm{h}$ to $10 \mathrm{~km} / \mathrm{h}$ & {$[10]$} \\
\hline
\end{tabular}

a The peak data rate is defined as the maximum data rate under ideal conditions (in bps), i.e., under error-free conditions and when all assignable radio resources for the corresponding link direction are utilized. The user experienced data rate is the $5 \%$ point of the Cumulative Distribution Function (CDF) of the user throughput.

$\mathrm{b}$ The maximum UE speed (in $\mathrm{km} / \mathrm{h}$ ) at which a defined Quality of Service (QoS) can be achieved.

discussed in [10], [11]. Some discussion related to how DC might assist in meeting these requirements is latter presented in Section V. However, before presenting this discussion, the next two sections present the architectures for DC and how the UEs might be connected to those new architectures.

\section{System ARchitectures for DuAl Connectivity}

In order to allow more flexible and cost-effective HetNet deployments, a new network architecture has been proposed and attracted a lot of attention during the standardization process of the LTE Release 12. In this architecture, there is a split between the Control and User Planes, where, basically, the Control Plane is responsible for transmitting system information and controlling the UE connectivity, and the User Plane (also referred as Data Plane) handles UE specific data [5]. Considering this separation, the Control and User Planes might not be transmitted by the same network node, which brings important new features that enable the DC, as explained in more details in the sequel.

Furthermore, this architecture allows network operators to be more flexible in the network management by, for example, designing the MeNBs to handle the UE connectivity and the SeNBs to be activated only when there is data to be transmitted [5], which would decrease SeNBs' power consumption.

\section{A. User Plane}

Considering the perspective of the User Plane, after an evaluation of several possible options, two DC solutions have been standardized by 3GPP: (i) the User Plane data is split in the Core Network $(\mathrm{CN})^{2}$, which corresponds to the

${ }^{2}$ We consider that the $\mathrm{CN}$, among other entities, is composed by the Mobility Management Entity (MME), which is responsible for the Control Plane mobility management, the Serving Gateway (S-GW) and Packet Data Network Gateway (P-GW), which routes and forwards the User Plane to the eNBs.
1A configuration, or (ii) the User Plane is split in the MeNB, which is known as the $3 \mathrm{C}$ configuration [3], [6], illustrated in Fig. 2. Besides these two configurations, legacy SC is also shown. The User Plane is composed by the following protocol layers: Packet Data Convergence Protocol (PDCP), Radio Link Control (RLC) and Medium Access Control (MAC) [6].

\section{User Plane: 1 A configuration}

This configuration is depicted in Fig. 2b. The number 1 means that the S1-U (i.e., the $\mathrm{S} 1$ interface $^{3}$ related to the User Plane) terminates at both MeNB and SeNB, and the letter A stands for independent PDCP layers, i.e., there are independent User Plane end points in both MeNB and SeNB.

The 1A configuration is in practice realized by the establishment of two types of radio bearers: (i) Master Cell Group (MCG) bearers and (ii) Secondary Cell Group (SCG) bearers. A MCG bearer is a radio bearer that is served only by the MeNB, as the Radio Bearer \#1 in Fig. 2b, while a SCG is a radio bearer served only by the SeNB, as the Radio Bearer \#2 in Fig. 2b. In order to support these bearers, both MeNB and SeNB need to have a S1-U termination. Some authors consider that in this case there is a data (bearer) split in the CN. Therefore, for these two types of bearers, when a radio bearer is configured, it can only be transmitted from or towards either the MeNB or the SeNB involved in the DC configuration [3].

Since both MeNB and SeNB have a S1-U link, an advantage of this configuration is that the MeNB does not need to buffer or process the packets that come from a bearer that is transmitted by the SeNB. However, a drawback is that a UE cannot utilize radio resources across the MeNB and SeNB for the same bearer. This situation is illustrated in Fig. 2b, where

\footnotetext{
${ }^{3}$ The $\mathrm{S} 1$ interface is a link between an eNB and an Evolved Packet Core (EPC), providing an interconnection point between the Evolved Universal Terrestrial Radio Access Network (E-UTRAN) and the EPC. It is also considered as a reference point.
} 


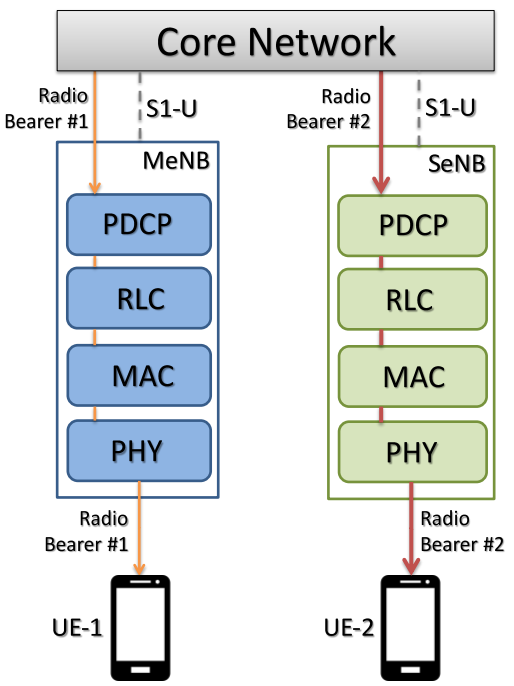

(a) Single connection.

Fig. 2. Radio protocol architecture for SC (legacy) and DC.

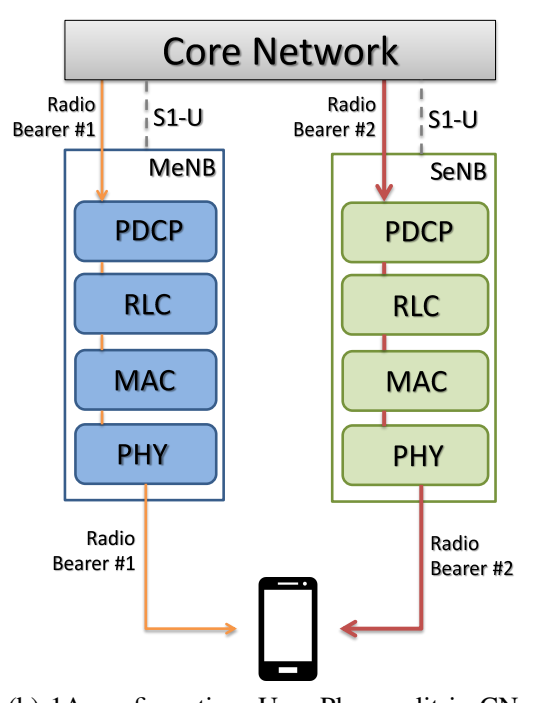

(b) $1 \mathrm{~A}$ configuration: User Plane split in $\mathrm{CN}$.

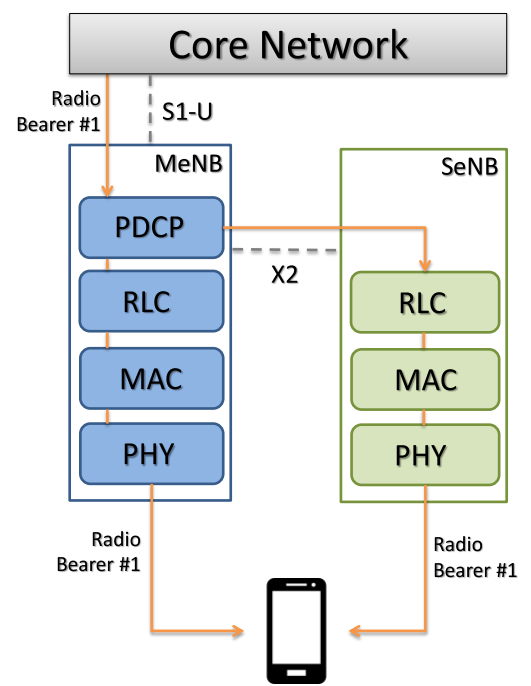

(c) 3C configuration: User Plane split in MeNB. albeit the UE is in DC with both MeNB and SeNB, the UE transmission of the radio bearers \#1 and \#2 are independent for the MeNB and SeNB. Therefore, the user throughput for a given application is not increased by the DC itself [6].

\section{User Plane: $3 C$ configuration}

This configuration is illustrated in Fig. 2c. The number 3 means that the S1-U terminates at the MeNB and bearer split is performed in the MeNB, thus there might exist a single bearer for each UE in DC and its flow split occurs in the MeNB. The letter $\mathrm{C}$ stands for independent RLC layers, i.e., there is a single PDCP layer located at the MeNB and two independent RLC layers in the MeNB and SeNB.

Most studies consider that the PDCP is implemented in the MeNB. However, there is also the option of moving the common PDCP layer to the $\mathrm{CN}$, being implemented in a new coordination entity that would play the role of a gateway for a group of LTE and NR eNBs in their coverage area [9].

Besides MCG bearers and SCG bearers, a third type of bearer can be established using the $3 \mathrm{C}$ configuration, the so-called split bearer. This type of bearer is characterized by a single flow that is transmitted from the $\mathrm{CN}$ to the PDCP protocol layer located at the MeNB. Then, the MeNB splits the traffic and forwards the packets to the MeNB RLC and/or the SeNB RLC [9]. The split bearer is exemplified by the radio bearer \#1 in Fig. 2c. Furthermore, this type of bearer allows the network operators to exploit the transmission diversity, where algorithms in the MeNB PDCP could be designed to either (i) forward the same packets to both MeNB RLC and SeNB RLC in order to enhance the system reliability, or (ii) forward different packets to MeNB RLC and SeNB RLC aiming to increase the per-user throughput.

Since there is only one S1-U termination at the MeNB in the $3 \mathrm{C}$ configuration, this entity needs to route, process and buffer all DC traffic, which is a drawback of this alternative. Furthermore, an additional feature needed for the $3 \mathrm{C}$ configuration is that there has to be a flow control between the MeNB and SeNB. On the other hand, the main benefit of this approach is that a single UE in DC might utilize radio resources across both MeNB and SeNB for the same bearer, thus increasing the user throughput for a given application, which is one of the requirements for $5 \mathrm{G}$ networks.

This main benefit of this alternative is illustrated in Fig. 2c, where the radio bearer \#1 is transmitted utilizing radio resources across both MeNB and SeNB. However, this comes at the cost of increasing the transport and processing capabilities in the MeNB.

\section{B. Control Plane}

In the LTE protocol architecture, the Radio Resource Control (RRC) layer is responsible for the Control Plane functions. Some specific functions performed in this layer are broadcast of reference signal and system information, configuration of lower layer protocols, mobility management, and measurement and configuration reporting. In the following, it is presented how the RRC would work in the DC scenarios.

\section{Control Plane: 1A configuration}

In the $1 \mathrm{~A}$ configuration, besides having independent User Plane stacks, the MeNB and SeNB also have independent Control Planes, i.e., independent RRC layers. Considering the UE mobility between SeNBs, this configuration presents some disadvantages that are not present in the $3 \mathrm{C}$ configuration, such as: (i) there has to be packet forwarding between the SeNBs, thus service interruption may be noticeable since the MeNB is unable to handle the SeNB bearers, and (ii) the UE mobility is not hidden to the $\mathrm{CN}$ since it is necessary to involve the MME in this process [6].

\section{Control Plane: $3 C$ configuration}

Regarding the $3 \mathrm{C}$ configuration for the DC technology, the assumption is that there is only one S1-MME connection per 
UE and this link is terminated at the MeNB. Since the RRC functions cited in the beginning of this subsection do not require synchronization with lower layer protocols, the authors in [8] have proposed a common RRC across multiple RATs, which allows the optimization of control functionalities in order to enhance the overall system performance. Considering the integration LTE-NR, this common Control Plane could be handled by the MeNB with the objective of providing a more robust system, thus the MeNB will be the entity responsible for the maintenance of the RRC connections. This implies that the MeNB controls the DC configuration: it is responsible for generating and sending all RRC messages to the UE. Consequently, the UE RRC entity receives all messages sent only from one entity, located at the MeNB, and the UE only responds back to that entity [6].

The transmission of RRC messages is not supported via the SeNB. Thus, if the SeNB needs to change or release its own part of the RRC configuration, it sends RRC messages to the MeNB via the X2 interface. Then, the MeNB transmits the RRC message to the UEs. The SeNB has its own pool of radio resources and is primarily responsible for allocating them to its connected UEs. Notice that some coordination between MeNB and SeNB over the X2 interface is needed in order to enable the optimization of resource management procedures [6].

Furthermore, the common Control Plane assumption would enable new features: (i) Control Plane diversity, where a UE in DC having a single control point would be able to switch links without explicit signaling, which increases reliability and (ii) fast Control Plane switching, where the UE would be able to be connected to a single control point via LTE or NR and switch very fast between them [8].

\section{USER CONnEctivity SOLUtions}

In Section III, the system architectures for scenarios with DC were presented. In this section, we discuss the possible user connectivity solutions considering the new system architectures for DC.

\section{A. Traditional Handover}

Considering the current scenario of cellular networks, the LTE technology supports handover inside the E-UTRAN and also to other legacy RATs (e.g., Universal Mobile Telecommunications System (UMTS), Global System for Mobile Communications (GSM)). In the LTE technology, there are two types of handover procedures: (i) a X2-based handover that is performed for intra-RAT handovers only, based on the interconnection between the source and target eNBs, and (ii) S1-based handover which is performed when there is no X2 interface between the eNBs or when the target eNB belongs to a different RAT [9].

The handover between RATs, also known as Hard Handover $(\mathrm{HH})$, typically occurs when the signal from the current RAT to which the UE is connected is below a certain threshold and the signal from a target RAT is above another threshold. Since this type of handover involves multiple RATs, there has to be some communication between the source RAT and the $\mathrm{CN}$ requesting the handover to the target RAT. This procedure causes a transmission interruption for the involved UE because there is a short gap of time where this UE is not connected to any of the RATs, which is the main disadvantage of the $\mathrm{HH}$. This procedure is illustrated in Fig. 3a considering the new architecture that allows Control and User Planes split.

\section{B. Dual Connectivity}

The new connectivity solution for the UEs is the DC, where the UE has both Control and User Planes simultaneously connected to both MeNB and SeNB. In order to benefit from the DC, a UE needs to have separate protocol stacks (RLC and MAC), one for the MeNB and another for the SeNB. Besides that, the UE needs to be equipped with dual radios with both receiver ( $\mathrm{Rx})$ and transmitter (Tx), thus allowing them to be fully connected to both MeNB and SeNB. This connectivity solution is illustrated in Fig. $3 b$.

This solution allows a User Plane aggregation, where a single UE can receive a single flow over multiple RATs (the $3 \mathrm{C}$ configuration) or different flows on different RATs (the 1A configuration) [8].

\section{Fast Switching}

There is another possible user connectivity solution that has arisen as a variant of the DC, which is known as Fast-RAT Scheduling (FS). In this solution, the UE would have a Control Plane connection established with two different RATs simultaneously and only one User Plane connection active to a given RAT, which can be rapidly switched between the RATs, as illustrated in Fig. 3c [8], [9]. Considering the current mobile network, this switch is only performed with a handover, which however requires a gap of time that might introduce a short period of service interruption.

Notice that in order to enable the possibility of this solution for a given UE, there should exist a robust and common Control Plane (common RRC) for both RATs [8]. Another point that is worth mentioning is that a UE equipped with a single radio (one $\mathrm{Tx}$ and $\mathrm{Rx}$ ) would be able to enjoy this solution since there would be only a single and common Control Plane and only one User Plane connection at a time.

\section{Radio Resource Management in DC Scenarios}

The LTE-NR scenario with DC imposes new challenges and requires innovative solutions regarding RRM techniques. Considering the DC scenario, some important RRM functionalities deserve more attention in order to ensure the proper utilization of these new connectivity solutions, namely: (i) UE-cell association, (ii) MeNB-SeNB interaction and (iii) resource allocation. Each of these functionalities is discussed in more details in this section, where we present the state-of-the-art works related to each functionality and how the use cases presented in Section II may benefit from the DC technology.

\section{A. UE-cell association}

The first aspect to be highlighted is the UE-cell association, which involves the decision of how to configure UEs with 


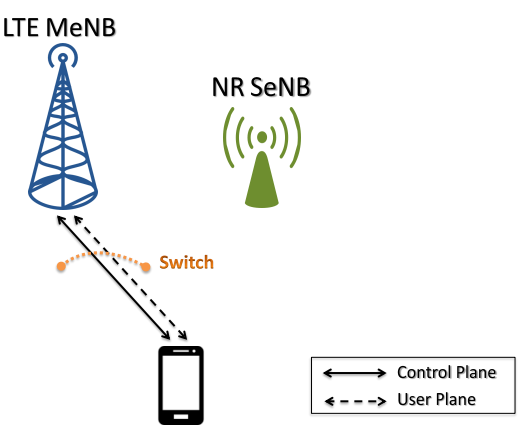

(a) Traditional handover.

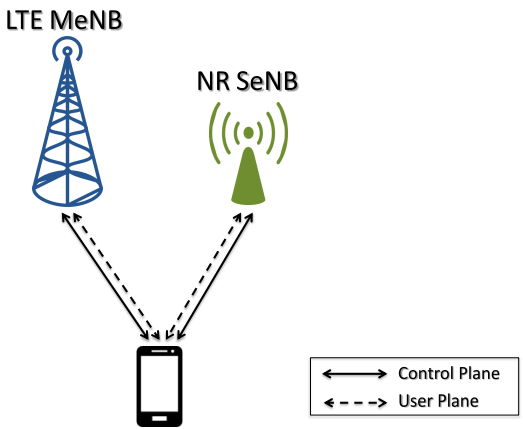

(b) Dual Connectivity (DC). Fig. 3. Possible user connections in multi-RAT scenarios composed by LTE and NR eNBs.

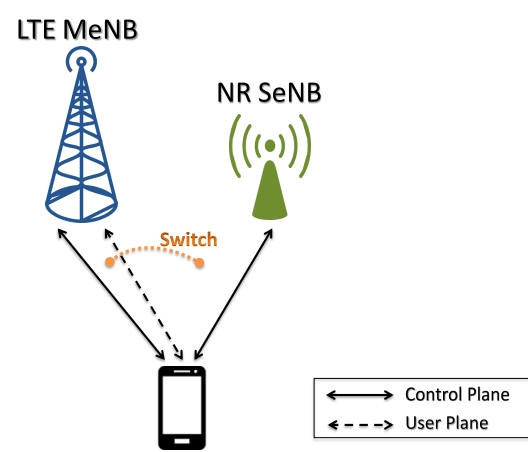

(c) Fast Switching (FS).
DC, FS, or even SC. In [12], the problem of RAT scheduling considering multi-RAT systems was studied, and the authors presented multiple mathematical formulations to model the network selection. However, the authors have not considered $\mathrm{DC}$ in their scenario.

Some approaches for RAT selection in a DC scenario using the radio quality measurements specified by 3GPP are presented in [6] and [13], but they have not considered QoS metrics during the RAT selection. In [14], the authors proposed a user-centric scheme where the users are responsible for performing the RAT selection according to criteria such as battery level, charges for using network services and delay in each network. However, even though they formulated their optimization problem considering the DC possibility, no performance evaluation was conducted in DC-enabled scenarios. In [15], the authors formulated an optimization problem for throughput maximization where the users had DC capabilities, while in [16] the authors considered a throughput maximization problem for cell selection where proportional fairness among users was taken into account, but the HetNet scenarios analyzed therein were composed of only LTE eNBs. In [17], an optimal user association algorithm considering fairness is proposed. The authors in [18] compared the amount of control signaling involved in network-controlled and UE autonomous mobility management for high-speed scenarios, but still the scenario did not consider the LTE-NR integration.

Considering the LTE-NR integration, which further complicates the problem due to intrinsic characteristics expected for the 5G channel [8], the authors in [19] proposed a DC protocol with the objective of allowing UEs to have uninterrupted connection to a LTE MeNB and switching the connection to NR SeNBs. In [20], the authors analyzed how to improve the QoS in LTE-NR multi-RAT scenarios by comparing different channel measurement metrics. The authors concluded that metrics related to signal quality should be prioritized over metrics only related to signal strength. As a consequence, when the system load increases, less users should stay in DC to limit the interference and increase the performance.

Therefore, one can notice that how to best perform the RAT selection in a LTE-NR scenario with DC needs still to be further investigated in the literature. For instance, since the target for mobility interruption time for the eMBB and URLLC is $0 \mathrm{~ms}$, UEs/devices from these use cases might first establish a connection with the MeNB before performing a handover between SeNBs, such as considered in [19]. Thus, the eMBB and URLLC UEs/devices would be connected to the more reliable connection provided by the LTE technology during the handover, so that there would not exist any interruption time. Furthermore, QoS metrics could be used coupled with channel quality measurement for the RAT selection, such that the user could be monitoring the quality of its two connections and decide if it is benefiting from both connections based on QoS and channel quality metrics. Another point is that UE autonomous mechanisms should be preferable compared to network-controlled ones because they demand less signaling, as demonstrated in [18].

\section{B. MeNB-SeNB interaction}

Another important point is the MeNB-SeNB interaction. Because of the DC technology, it is inevitable, either in the $1 \mathrm{~A}$ or $3 \mathrm{C}$ configuration, to have communication between the MeNB and SeNB via X2 interface to support their interaction in the RRM and power control [6]. Furthermore, in the 3C configuration, an efficient flow control mechanism must exist in the MeNB to determine the amount of data that should be forwarded to the SeNB without overloading it or leaving the SeNB without data to be transmitted.

Regarding the flow control mechanism for the DL in the $3 \mathrm{C}$ configuration, some studies are available in the literature. In [21], a mechanism is proposed where there is a fixed percentage of data that the MeNB sends to the SeNB. Dynamic mechanisms based on the SeNB radio capacity and backhaul latency or MeNB buffer status and radio capacity are proposed in [22] and [13], respectively. In [23], a scheme of flow control and traffic scheduling is designed as a Mixed Integer Linear Programming (MILP) aiming at maximizing the network throughput. The authors in [24] proposed a flow control mechanism based on a constrained Semi-Markov Decision Problem (SMDP) with the objective of minimizing the average delay in the system. However, the works in [23] and [24] only analyzed the optimal solution to their optimization problem; the authors of [23] and [24] did not propose a tractable suboptimal solution. All the works mentioned in this paragraph considered LTE-based HetNet scenarios. 
The authors in [25] formulated an optimization problem for flow control with the objective of minimizing the power consumption at the MeNB and SeNBs considering QoS requirements for each UE. The scenario analyzed therein was composed of Non-Orthogonal Multiple Access (NOMA)-enabled MeNB and SeNB. Considering the integration between LTE and Wi-Fi, the works in [26], [27] proposed flow control mechanisms with fairness considerations.

Nevertheless, as far as we know, there is no work in the literature considering the flow control in the LTE-NR scenario with DC. This scenario poses new challenges due to the requirements imposed by the eMBB, URLLC and mMTC use cases, as shown in Section II.

The URLLC services are expected to have latency requirements of $0.5 \mathrm{~ms}$ [11] or $1 \mathrm{~ms}$ [10]. Therefore, due to this strict requirement, considering the $3 \mathrm{C}$ configuration, it is not always recommended to send data from URLLC services over the non-ideal backhaul because its latency is usually higher than $1 \mathrm{~ms}$ [4]. On the other hand, due to high reliability requirements of URLLC services, the flow control algorithm in the $3 \mathrm{C}$ configuration can be designed to forward the same packets to both MeNB RLC and SeNB RLC in order to enhance the system reliability for these services. Considering the eMBB applications, the flow control algorithm in the 3C-configuration should send different packets to MeNB RLC and SeNB RLC to meet the rigorous requirements of data rate from these applications. Furthermore, in the 1A configuration, the eNBs serving eMBB applications to UEs in DC could communicate aiming at increasing the per-user throughput of these services.

Thus, flow control algorithms aiming at, for instance, improving the load balancing between RATs, giving more priority to a given service or maximizing the system capacity are still to be designed for the LTE-NR scenario with DC.

\section{Resource Allocation}

Finally, the last step would be the resource allocation, which involves the problem of how to schedule UEs with or without DC. In [28], several scheduling algorithms for DL traffic are discussed, but these schemes cannot be applied directly to the DC scenario because they consider a single RAT. A new version of the Proportional Fair (PF) scheduling algorithm is presented in [29], which was modified to consider the throughput of the DC UEs over MeNB and SeNB during the resource allocation. This modification allowed the PF to maintain its property of providing a good trade-off between user fairness and system capacity. The authors in [30] studied a cost minimization problem considering per-user minimum rate constraints for the UL of DC-enabled scenarios, where the authors proposed optimal rate and power allocation schemes. In [31], a resource allocation optimization problem was proposed for computing the user association and frequency slots allocation with the objective of rate maximization. Considering a DC-enabled scenario with mmW backhaul links connecting the LTE eNBs, the authors in [32] proposed an optimization problem considering a utility function that can be configured to operate as max-min fairness or proportional fairness policies. In [33], the authors have considered that eNBs could eavesdrop UE's data when the information is transmitted via backhaul link and then proposed a resource allocation scheme to guarantee secrecy of information and minimize the power consumption and resource usage.

However, to the best of our knowledge, no scheduling algorithm has been proposed for the LTE-NR scenario. For this specific scenario, the algorithm to be designed should be able to guarantee the strict delay requirements and high throughput demands of the eMBB and URLLC use cases of the upcoming $5 \mathrm{G}$ networks [10], which could be accomplished by exploiting the different numerologies provided by the NR technology [34]. For example, as soon as the packets from URLLC services arrive, they should be transmitted in order to not exceed the delay target of $0.5 \mathrm{~ms}$ or $1 \mathrm{~ms}$. In order to meet such strict requirements, a smaller Transmission Time Interval (TTI) duration expected for the NR radio technology would be very useful. For eMBB services, the preference would be to transmit a high amount of data over the RATs to fulfill the throughput demands.

\section{PERFormance EVAluATion}

This section presents a performance evaluation considering the bearer split configuration of the DC connectivity technology in multi-RAT scenarios composed of LTE and NR eNBs. Firstly, the simulation assumptions along with the proposed resource allocation solution are presented in Section VI-A and VI-B, respectively, and then the simulation results and discussion are shown in Section VI-C.

\section{A. Simulation Assumptions}

The simulation environment adopted in the present work is aligned with the $3 \mathrm{C}$ configuration (see Section III) presented in the 3GPP specifications in [6], [35]. The 1A configuration was not simulated mainly because its specification is still in progress in Release 15 of the $5 \mathrm{G}$ specifications by $3 \mathrm{GPP}$. Notice that for simulating the 1A configuration, we would need to have the 5G BSs connecting to a legacy $\mathrm{CN}$, which still requires some investigation and standardization before modeling it in a computer simulator. Another option would be to connect the $4 \mathrm{G}$ and $5 \mathrm{G}$ BSs to a $5 \mathrm{G} \mathrm{CN}$, which also requires some further studies to define how the connection between $4 \mathrm{G}$ BSs and the $5 \mathrm{G} \mathrm{CN}$ is going to work. Besides that, the first deployment of $5 \mathrm{G}$ BSs is predicted to be in the form of the Non-Stand-Alone (NSA) 5G, where 5G BSs are anchored at the $4 \mathrm{G} \mathrm{BSs,} \mathrm{which} \mathrm{follows} \mathrm{the} \mathrm{bearer} \mathrm{split} \mathrm{configuration}$ that is based on the $3 \mathrm{C}$ configuration. Therefore, given these reasons, this paper concentrates on the performance evaluation considering only the $3 \mathrm{C}$ configuration, as also done by [18], [19], [23], [32], [36], for example.

The network topology is comprised of an hexagonal grid of three-sector macro LTE eNBs, which are the MeNBs in our scenario. Then, one micro NR eNB, which is the SeNB, is randomly deployed on each sector of the LTE eNBs [11]. This deployment is illustrated in Fig. 4. The UEs dropping criterion follows a hotspot UE distribution per sector of the LTE MeNB, where $75 \%$ are deployed within the NR SeNB coverage area 
and the other $25 \%$ are uniformly deployed within the LTE MeNB sector. The NR SeNB coverage area is defined by the region where the user measures a signal strength above a given threshold, which is explained during the handover description.

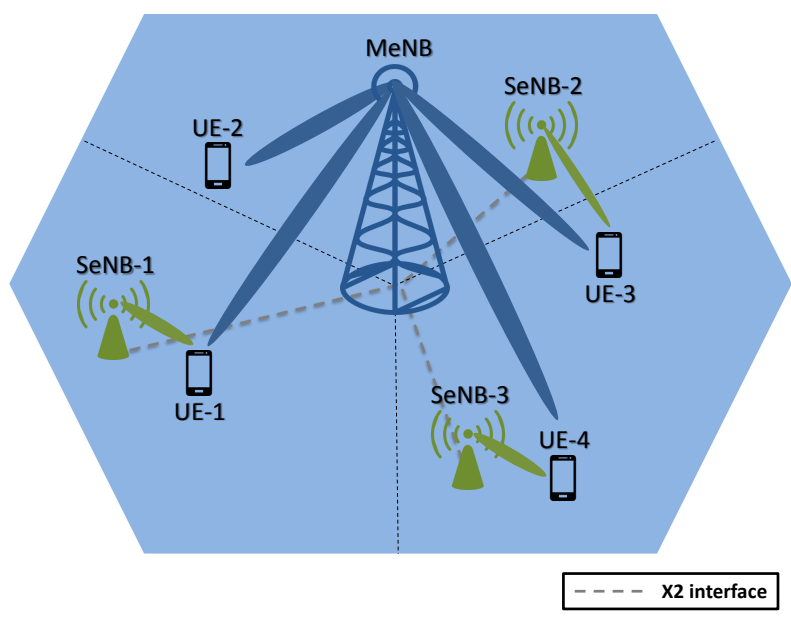

Fig. 4. Network topology adopted for the simulations. A LTE MeNB is positioned at the center of a three-sector hexagonal grid and one NR SeNB is randomly deployed per sector.

Each UE is configured to have one bearer and the traffic type of the bearer is a Constant Bit Rate (CBR) flow. The UEs are considered satisfied if their total throughput is higher than or equal to a throughput requirement of $20 \mathrm{Mbps}$.

All the results are evaluated as a function of the number of UEs per MeNB sector, which includes the UEs in DC and in SC. Furthermore, all the results are presented with the $95 \%$ bootstrap confidence interval of the mean of the samples. A summary of the simulation parameters are presented in Tables II and III.

The handover procedure follows the handover events and metrics described by 3GPP in [6], [42] and widely used in the literature, such as in [13], [18]. In our simulations, we consider that all handover events are triggered based on Reference Signal Received Power (RSRP) measurements [6], [42]. On one hand, the handover between MeNBs are usually triggered by the A3 event, which happens when a neighboring cell becomes offset better than the current serving cell. On the other hand, the handover between SeNBs is triggered by the A6 event, which happens when the neighboring small cell becomes offset better than the current serving small cell. For a user who is in SC with a MeNB, a SeNB addition happens based on the A4 event, which is when the neighboring small cell becomes better than a certain threshold, which defines the NR SeNB coverage area. On the other hand, for UEs in DC, a SeNB release occurs when the A2 event is triggered, i.e., when the serving small cell signal becomes worse than a given threshold.

The baseline flow control algorithm analyzed herein was discussed in [21], where a fixed percentage, $x \%$, of data is sent by the MeNB to the SeNB via the backhaul link, and another fixed percentage $(100-x) \%$ is sent by the MeNB to the UE. As mentioned in section III, this flow control algorithm runs at the PDCP layer. In the figures presenting the results and in the results discussion, we refer to the baseline algorithm as
$\mathrm{F}(x)$, where $x$ is the percentage of data sent by the MeNB to the UEs.

The resource allocation algorithms considered herein are employed for all LTE and NR base stations. These algorithms are executed based on a cross-layer interaction between MAC and physical layers. Regarding resource allocation algorithms for benchmarking, as mentioned in Section V-C, the algorithms described in [28] would not be suitable for applications in DC-enabled scenarios because they consider only single connectivity in their formulation. Thus, it would not be fair to use those algorithms for comparison. Besides that, the performance of the algorithms from [28] was beaten in previous studies by the traditional PF algorithm [43], [44], which is used as the baseline for the algorithm used as benchmarking in this study. Therefore, only the cross-carrier PF [13] was used for comparison, which is a modified version of the well-known PF algorithm that attempts to guarantee fairness in scenarios with DC by modifying the scheduling metric. Each BS $b$ selects the user $j^{\star}$ to transmit on RB $k$ at TTI $n$ according to

$$
j_{k, b}^{\star}[n]=\underset{j \in \mathcal{J}_{b}}{\arg \max }\left\{\frac{r_{j, k, b}[n]}{\sum_{b \in \mathcal{B}} T_{j, b}[n-1]}\right\},
$$

where $r_{j, k, b}[n]$ is the achievable rate of user $j$ on RB $k$ of BS $b$ and $\sum_{b \in \mathcal{B}} T_{j, b}[n-1]$ is the user throughput considering all previous and current connections of user $j$ to all BSs $b \in \mathcal{B}$ up to TTI $n-1$, i.e, this is the total user throughput considering the DC.

\section{B. Proposed resource allocation scheme}

Besides the cross-carrier PF algorithm, another resource allocation scheme was considered during the performance evaluation, which is a contribution of the present work. The proposed resource allocation solution is an extension of the Throughput-based Satisfaction Maximization (TSM) algorithm [43], which is a utility-based algorithm that was originally proposed for users in SC. The proposed solution running on $\mathrm{BS} b$ selects the user $j^{\star}$ to transmit on $\mathrm{RB} k$ at TTI $n$ according to

$$
j_{k, b}^{\star}[n]=\underset{j \in \mathcal{J}_{b}}{\arg \max }\left\{U^{\prime}\left(\sum_{b \in \mathcal{B}} T_{j, b}[n-1]\right) \cdot r_{j, k, b}[n]\right\},
$$

where $U(\cdot)$ is a sigmoidal function given by [44]:

$$
U\left(\sum_{b \in \mathcal{B}} T_{j, b}[n-1]\right)=\frac{1}{1+e^{\mu\left(\sum_{b \in \mathcal{B}} T_{j, b}[n-1]-T_{j}^{\mathrm{req}}\right) / \sigma}},
$$

where $T_{j}^{\text {req }}$ is the throughput requirement of the user, $\mu=-1$ yielding an ascending utility function and $\sigma=0.1088$, which is a parameter that controls the regulation of the logistic function shape [44]. The modification we propose herein compared to the original TSM is to use the total user throughput, as done in the cross-carrier PF, so that we capture the global QoS experience of the users, instead of only the local experience. This modification relies on the 
TABLE II

SIMULATION PARAMETERS FOR LTE AND NR

\begin{tabular}{|c|c|c|c|}
\hline Parameter & LTE & NR & Ref.'s ${ }^{a}$ \\
\hline Layout & Macro layer: 1 hexagonal site with 3 sectors & $\begin{array}{l}\text { Micro layer: } 1 \text { randomly dropped NR BS per LTE } \\
\text { sector }\end{array}$ & {$[11]$} \\
\hline Scenario & 3GPP 3D Urban Macro & 3GPP 3D Urban Micro & [37] \\
\hline Inter-site distance & $1000 \mathrm{~m}$ & - & {$[37]$} \\
\hline BS height & $25 \mathrm{~m}$ & $10 \mathrm{~m}$ & [37], [38] \\
\hline Carrier frequency & $3.5 \mathrm{GHz}$ & $28 \mathrm{GHz}$ & {$[37],[38]$} \\
\hline System bandwidth & $20 \mathrm{MHz}$ & $100 \mathrm{MHz}$ & [37], [38] \\
\hline Subcarrier spacing & $15 \mathrm{kHz}$ & $60 \mathrm{kHz}$ & [39], [34] \\
\hline Num. of RBs & 100 & 125 & [40], [34] \\
\hline TTI duration & $1 \mathrm{~ms}$ & $0.25 \mathrm{~ms}$ & [39], [34] \\
\hline Num. of subcarriers per RB & 12 & 12 & [39], [34] \\
\hline Num. of OFDM symbols per RB & 14 & 14 & [39], [34] \\
\hline Noise figure & $9 \mathrm{~dB}$ & $9 \mathrm{~dB}$ & [38] \\
\hline BS Tx power & $49 \mathrm{dBm}$ & $35 \mathrm{dBm}$ & {$[38],[38]$} \\
\hline Tx antenna type & Uniform linear array with 8 elements & Uniform rectangular array $4 \times 4$ & [38] \\
\hline Tx antenna element pattern & 3GPP 3D & 3GPP 3D & [37] \\
\hline Offset for handover & $3 \mathrm{~dB}$ & $3 \mathrm{~dB}$ & {$[18]$} \\
\hline Minimum RSRP for connection & $-100 \mathrm{dBm}$ & $-90 \mathrm{dBm}$ & \\
\hline
\end{tabular}

${ }^{a}$ Whenever two references appear, the first refers to LTE and the second to NR. Also, only one reference refers to both RATs.

TABLE III

COMMON SIMULATION PARAMETERS FOR BOTH RATS.

\begin{tabular}{lll}
\hline Parameter & Value & Ref.'s \\
\hline Link adaptation & Link level curves ${ }^{\mathrm{a}}$ & {$[41]$} \\
Power allocation & Equal power allocation & \\
UE distribution & Uniform in the macro area & \\
UE height & $1.5 \mathrm{~m}$ & \\
UE speed & $3 \mathrm{~km} / \mathrm{h}$ & \\
UE bearer type & $\mathrm{CBR}$ & \\
UE throughput requirement & $20 \mathrm{Mbps}$ & \\
Confidence interval & $95 \%$ & \\
\hline
\end{tabular}

${ }^{a}$ For a BLock Error Rate (BLER) of $10^{-4}$.

b $75 \%$ in hotspot (within NR BS coverage area).

communication between MeNB and SeNB through backhaul link, which is aligned with the agreements in [6] and has already been used in the cross-carrier PF [13]. For simplicity, in the remaining of this article, we will refer to the cross-carrier PF algorithm just as PF. Also, the proposed resource allocation is refered to as just TSM.

\section{Simulation Results}

Since the handover procedure used in all cases is equal, the only differences between the compared algorithms are in the flow control and resource allocation algorithms. Thus, we differentiate the curves just by the percentage of traffic that is sent by MeNB and SeNB, which is related to the flow control algorithm, and the resource allocation technique employed. Regarding the flow control algorithm, the values used for comparison are the same used in [23], which are $\mathrm{F}(30), \mathrm{F}(50)$ and $F(70)$. These values are sufficient to capture the important behaviors of the performance analyses, such as the verification of the transmission capacity of each RAT, which connection should be used to forward the data of users in SC and DC, and the importance of the resource allocation algorithms when the FCA is not operating in its optimal point. More details about the captured behaviors are presented during the results discussion.
The percentage of satisfied users is the first performance metric we investigate. Fig. 5 presents the percentage of satisfied UEs when the number of UEs increases. One can observe that the best performance is achieved by the $\mathrm{F}(30)+\mathrm{TSM}$ and $\mathrm{F}(50)+\mathrm{TSM}$. Comparing the curves $\mathrm{F}(30)+\mathrm{TSM}$ and $\mathrm{F}(30)+\mathrm{PF}$, the handover procedure and flow control algorithm are equal, such that the gains obtained come only from the proposed resource allocation. The gains occur because the proposed solution aims at maximizing the user satisfaction by allocating the resources based on their QoS level using a well-designed utility function [44].

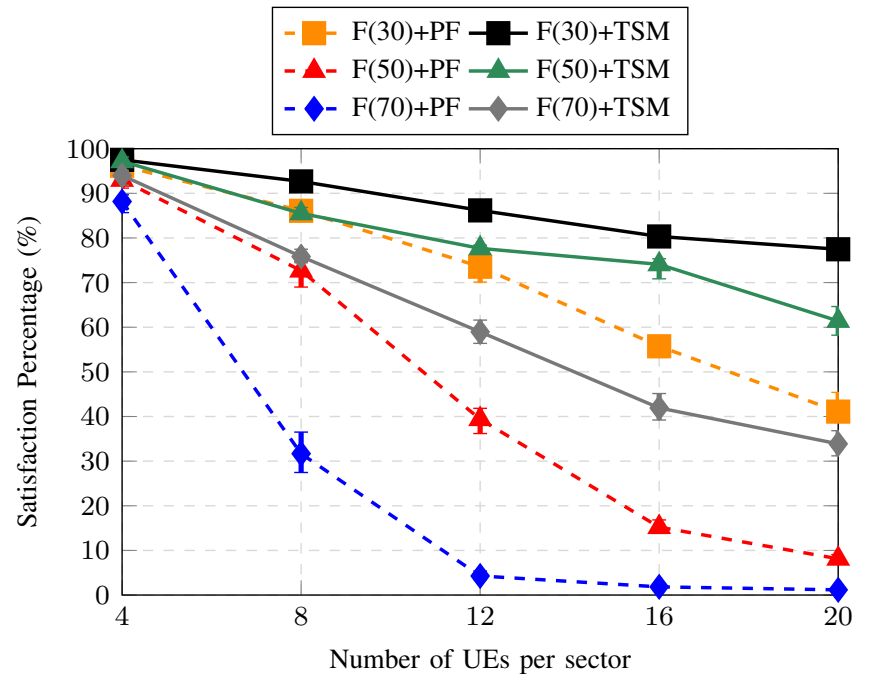

Fig. 5. Percentage of satisfied UEs when the system load increases.

It is important to mention that the highest performance obtained by both TSM and PF happened when the flow control algorithm was $\mathrm{F}(30)$, i.e., when the MeNB transmitted only $30 \%$ of the data to each user and sent the other $70 \%$ to the SeNB. Notice that, regardless of the resource allocation algorithm, when the percentage of data sent to the MeNB increases, the performance decreases. Consequently, higher satisfaction values are obtained by $\mathrm{F}(30)$ when combined to a 
given resource allocation algorithm. The reason behind that is the higher transmission capacity of the SeNB that is employing the NR RAT, which has a higher bandwidth and shorter TTI. However, notice also that the curve $\mathrm{F}(50)+\mathrm{TSM}$ presented very similar or higher performance than the $\mathrm{F}(30)+\mathrm{PF}$, showing the importance of a well-designed resource allocation algorithm even when the flow control algorithm is not at its best.

Fig. 6 depicts the total system throughput when the number of UEs in the system increases. Notice again that the combination $\mathrm{F}(30)+\mathrm{TSM}$ achieved the best results. This means that the proposed resource allocation algorithm not only is able to maximize the user satisfaction, but it is also capable of providing the highest system throughput values. Also, one can see that differently from what is observed in Fig. 5, in Fig. 6 the curve $F(50)+T S M$ presented lower values of total system throughput if compared to the $\mathrm{F}(30)+\mathrm{PF}$. This behavior is explained by the fact that the flow control in $\mathrm{F}(30)+\mathrm{PF}$ sends more data to the SeNB, whose higher transmission capacity provides better values of system throughput.

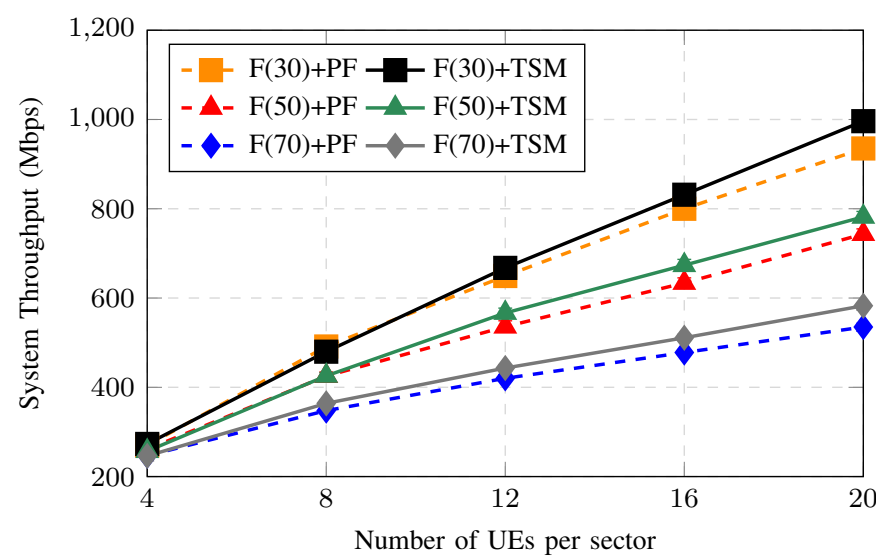

Fig. 6. Total system throughput.

In Fig. 7, the mean user throughput values considering all UEs in the system are presented. The best performance obtained by the $\mathrm{F}(30)+\mathrm{TSM}$ in the user satisfaction percentage (Fig. 5) is a consequence of the results presented in Fig. 7. One can see that even for high system loads, the mean throughput provided by $\mathrm{F}(30)+\mathrm{TSM}$ is always above the throughput requirement of $20 \mathrm{Mbps}$. This is reflected in Fig. 5 where the percentage of satisfied users is always kept as high as possible.

Notice, however, that for low system loads, the F(30)+PF achieves slightly higher mean throughput values than the $\mathrm{F}(30)+\mathrm{TSM}$. This happens because the proposed resource allocation algorithm attempts to maximize the number of satisfied users, which does not mean maximizing the mean throughput of the users in the system. Notice that the policy of allocating radio resources based on a ratio between channel quality and throughput (see eq. (1)) guarantees a higher mean throughput only for low system loads, but the user satisfaction for all loads and mean throughput for high loads is deteriorated.

Since the two best performances presented in Fig. 5, Fig. 6 and Fig. 7 were always obtained by $\mathrm{F}(30)+\mathrm{TSM}$ and $\mathrm{F}(30)+\mathrm{PF}$, the final analysis is conducted only for these two solutions.

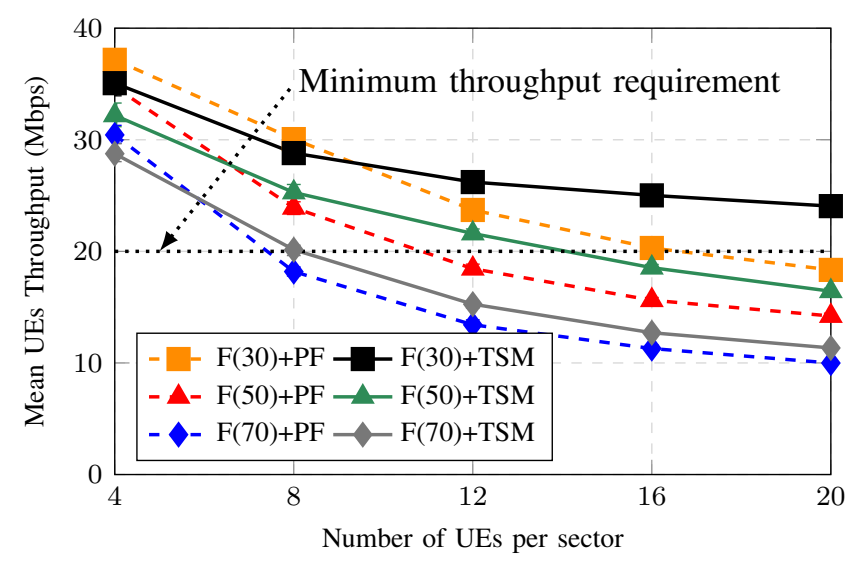

Fig. 7. Mean throughput of UEs.

Fig. 8 depicts the user satisfaction considering that the UEs in the system were separated into two groups: UEs in DC and UEs in SC. The gains provided by the F(30)+TSM are considerably high considering the group of users in DC; up to the system load we evaluated, all users in DC had their throughput requirement satisfied. Notice that best performance for the group of users in DC is also reflected for the group of users in SC. Fig. 8 shows that the overall user satisfaction maximization obtained by $\mathrm{F}(30)+\mathrm{TSM}$ in Fig. 5 is a consequence of maximizing the satisfaction of both groups of users.

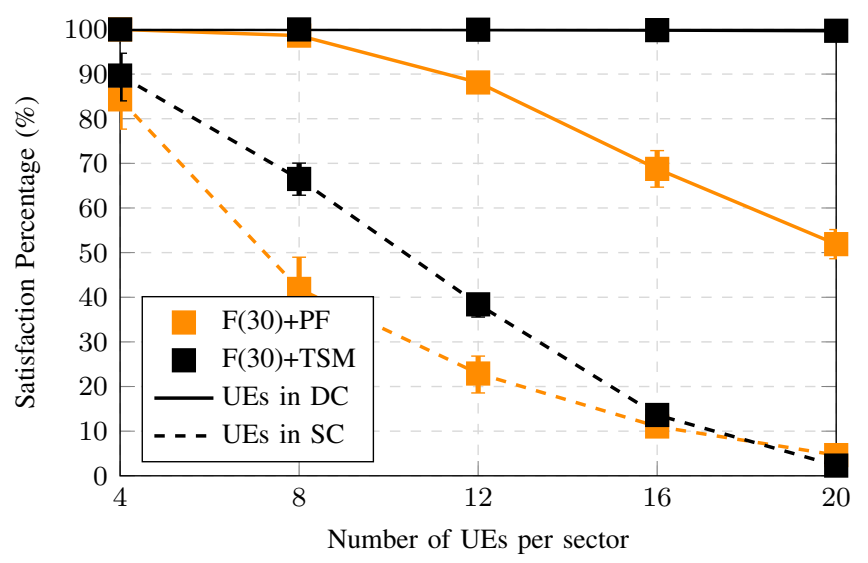

Fig. 8. Percentage of user satisfaction separating the UEs in DC and in SC.

\section{COnClusions AND PERspectives}

This work presents a comprehensive overview of the DC technology for $5 \mathrm{G}$ heterogeneous and multi-RAT scenarios composed of LTE and NR base stations, including possible options of architectures and a comprehensive survey regarding RRM in such scenarios. Furthermore, we propose an extension of a resource allocation scheme for DC scenarios and demonstrated the gains it provides by means of simulations in realistic $5 \mathrm{G}$ multi-RAT scenarios. It is worth mentioning that the gains demonstrated herein come only from the resource allocation proposal since we adopted existing handover procedures and flow control algorithm, which shows the importance of well-designed resource allocation algorithms 
for DC scenarios. Additionally, during the performance evaluation, it was observed that the higher is the percentage of data sent by the LTE MeNB, the lower is the satisfaction levels we obtain.

As futures work, one could look at developing algorithms for handover, flow control and resource allocation with common objectives such that the highest possible performance improvements are extracted from the bearer split configuration. Some directions on how to develop such a complete framework have been discussed throughout the present work.

The concepts and nomenclature presented in this work are based on the Release 12 by 3GPP, more specifically on the document [6]. However, the NR standardization process is ongoing [45], thus some new concepts may arise and the nomenclature might be modified. In fact, in [35] some new entities are been discussed, such as: a new eNB, named gNode $\mathrm{B}$ (gNB), which supports NR and connectivity to the Next Generation Core Network (NGC); the gNBs and the legacy LTE eNBs compose the New Radio Access Network (RAN); a new network core, named NGC; and the links between the entities in the New RAN (gNBs and LTE eNBs) are connected to the NGC by means of a new interface, named as NG. Furthermore, new possible configurations are presented in [35], such as a split bearer in the SeNB. Therefore, future works involving the DC technology should rely on the 3GPP specifications of Release 14 and beyond.

\section{REFERENCES}

[1] R. P. Antonioli, G. C. Parente, C. F. e Silva, E. B. Rodrigues, T. F. Maciel, and F. R. P. Cavalcanti, "Dual connectivity for LTE-NR cellular networks," in Proc. of the Brazilian Telecommun. Symp. (SBrT), São Pedro, Brazil, Sep. 2017, pp. 1-5.

[2] Cisco, "Cisco visual networking index: Global mobile data traffic forecast update, 2015-2020," White paper, Feb. 2016.

[3] C. Rosa, K. Pedersen, H. Wang, P. H. Michaelsen, S. Barbera, E. Malkamaki, T. Henttonen, and B. Sebire, "Dual connectivity for LTE small cell evolution: Functionality and performance aspects,' IEEE Commun. Mag., vol. 54, no. 6, pp. 137-143, Jun. 2016, ISSN: 0163-6804. DOI: 10.1109/MCOM.2016.7498101.

[4] 3GPP, "Scenarios and requirements of LTE small cell enhancements for E-UTRA and E-UTRAN," 3rd Generation Partnership Project (3GPP), TR 36.932, Dec. 2015, v.13.3.0.

[5] A. Zakrzewska, D. López-Pérez, S. Kucera, and H. Claussen, "Dual connectivity in LTE HetNets with split control- and user-plane," in 2013 IEEE Globecom Workshops, Dec. 2013, pp. 391-396. DOI: 10. 1109/GLOCOMW.2013.6825019.

[6] 3GPP, "Study on small cell enhancements for E-UTRA and E-UTRAN - higher layer aspects," 3rd Generation Partnership Project (3GPP), TR 36.842, Dec. 2013, v.12.0.0.

[7] METIS-II, "Draft overall 5G RAN design,” Deliverable 2.2, Jul. 2016. [Online]. Available: https://metis-ii.5g-ppp.eu/wp-content/uploads/ METIS-II_D2.2_V1.0.pdf (visited on 11/01/2016).

[8] I. D. Silva, G. Mildh, J. Rune, P. Wallentin, J. Vikberg, P. Schliwa-Bertling, and R. Fan, "Tight integration of new 5G air interface and LTE to fulfill 5G requirements," in Proc. of the IEEE Vehic. Tech. Conf. (VTC), May 2015, pp. 1-5. DOI: $10.1109 /$ VTCSpring.2015.7146134

[9] M. Polese, "Performance comparison of dual connectivity and hard handover for LTE-4G tight integration in mmWave cellular networks," Master's thesis, Department of Information Engineering, University of Padova, Padova, Italy, Jul. 2016.

[10] ITU, Draft new report ITU-R M.[IMT-2020.TECH PERF REQ] minimum requirements related to technical performance for IMT-2020 radio interface(s), ITU-R SG05 Contribution 40, Feb. 2017.

[11] 3GPP, "Study on scenarios and requirements for next generation access technologies," 3rd Generation Partnership Project (3GPP), TR 38.913, Aug. 2017, v.14.3.0. [Online]. Available: http : //www.3gpp . org/ DynaReport/38913.htm (visited on 08/03/2017).
[12] L. Wang and G. S. G. S. Kuo, "Mathematical modeling for network selection in heterogeneous wireless networks - a tutorial," IEEE Commun. Surveys Tuts., vol. 15, no. 1, pp. 271-292, 2013. DOI: 10. 1109/SURV.2012.010912.00044.

[13] H. Wang, C. Rosa, and K. I. Pedersen, "Dual connectivity for LTE-advanced heterogeneous networks," Wireless Networks, vol. 22, no. 4, pp. 1315-1328, 2016. DOI: 10.1007/s11276-015-1037-6.

[14] A. Awad, A. Mohamed, and C. F. Chiasserini, "Dynamic network selection in heterogeneous wireless networks: A user-centric scheme for improved delivery," vol. 6, no. 1, pp. 53-60, Jan. 2017. DOI: 10. 1109/MCE.2016.2614419.

[15] Y. Shi, H. Qu, and J. Zhao, "Dual connectivity enabled user association approach for max-throughput in the downlink heterogeneous network," Wireless Personal Commun., vol. 96, no. 1, pp. 529-542, 2017. DOI: 10.1007/s11277-017-4182-7.

[16] Q. Han, B. Yang, C. Chen, and X. Guan, "Matching-based cell selection for proportional fair throughput boosting via dual-connectivity," in Proc. of the IEEE Wireless Commun. and Networking Conf. (WCNC), IEEE, Mar. 2017, pp. 1-6. DOI: 10.1109/WCNC.2017.7925571

[17] N. Prasad and S. Rangarajan, "Exploiting dual connectivity in heterogeneous cellular networks," in Internat. Symp. on Modeling and Optim. in Mob., Ad Hoc, and Wireless Networks and Workshops, May 2017, pp. 1-8. DOI: 10.23919/WIOPT.2017.7959889.

[18] L. C. Gimenez, P. H. Michaelsen, and K. I. Pedersen, "UE autonomous cell management in a high-speed scenario with dual connectivity," in Proc. of the IEEE Personal, Indoor and Mob. Radio Commun. (PIMRC), IEEE, Sep. 2016, pp. 1-6. DOI: 10.1109/PIMRC. 2016. 7794951.

[19] M. Polese, M. Giordani, M. Mezzavilla, S. Rangan, and M. Zorzi, "Improved handover through dual connectivity in 5G mmwave mobile networks," IEEE J. Sel. Areas Commun., vol. 35, no. 9, pp. 2069-2084, Sep. 2017. DOI: 10.1109/JSAC.2017.2720338.

[20] V. F. Monteiro, M. Ericson, and F. R. P. Cavalcanti, "Fast-RAT scheduling in a 5G multi-RAT scenario," IEEE Commun. Mag., vol. 55, no. 6, pp. 79-85, Jun. 2017. DOI: 10.1109/MCOM.2017.1601094.

[21] 3GPP, 3GPP contribution R2-132859: Throughput evaluation and comparison of with and without UP bearer split, Aug. 2013.

[22] - 3GPP contribution R2-132833: Performance evaluation of user throughput enhancement with multi-stream aggregation over non-ideal backhaul, Aug. 2013.

[23] M. S. Pan, T. M. Lin, C. Y. Chiu, and C. Y. Wang, "Downlink traffic scheduling for LTE-A small cell networks with dual connectivity enhancement," IEEE Commun. Lett., vol. 20, no. 4, pp. 796-799, Apr. 2016, ISSN: 1089-7798. DOI: 10.1109/LCOMM.2016.2522404.

[24] P. K. Taksande, A. Roy, and A. Karandikar, "Optimal traffic splitting policy in LTE-based heterogeneous network," arXiv preprint arXiv:1710.11453, 2017

[25] Y. Wu and L. P. Qian, "Energy-efficient NOMA-enabled traffic offloading via dual-connectivity in small-cell networks," IEEE Commun. Lett., vol. 21, no. 7, pp. 1605-1608, Jul. 2017, ISSN: 1089-7798. DOI: 10.1109/LCOMM.2017.2685384.

[26] B. Jin, S. Kim, D. Yun, Y. Yi, H. Lee, and W. Kim, "Aggregating LTE and Wi-Fi: Fairness and split-scheduling," in Internat. Symp. on Modeling and Optim. in Mob., Ad Hoc, and Wireless Networks and Workshops, IEEE, May 2016, pp. 1-8. DOI: 10.1109/WIOPT.2016. 7492936

[27] S. Singh, M. Geraseminko, S.-p. Yeh, N. Himayat, and S Talwar, "Proportional fair traffic splitting and aggregation in heterogeneous wireless networks," IEEE Commun. Lett., vol. 20, no. 5, pp. 1010-1013, May 2016, ISSN: 1089-7798. DOI: 10.1109/LCOMM. 2016.2547418

[28] F. Capozzi, G. Piro, L. A. Grieco, G. Boggia, and P. Camarda, "Downlink packet scheduling in LTE cellular networks: Key design issues and a survey," IEEE Commun. Surveys Tuts., vol. 15, no. 2, pp. 678-700, May 2013, ISSN: 1553-877X. DOI: 10.1109/SURV.2012. 060912.00100

[29] Y. Wang, K. I. Pedersen, T. B. Sorensen, and P. E. Mogensen, "Utility maximization in LTE-advanced systems with carrier aggregation," in Proc. of the IEEE Vehic. Tech. Conf. (VTC), May 2011, pp. 1-5. DOI: 10.1109/VETECS.2011.5956494.

[30] Y. Wu, Y. He, L. Qian, and X. S. Shen, "Traffic scheduling and power allocations for mobile data offloading via dual-connectivity," in Proc. of the IEEE Internat. Conf. on Commun. (ICC), May 2016, pp. 1-6. DOI: $10.1109 /$ ICC.2016.7511339.

[31] G. Kato and T. Tachibana, "Resource allocation with optimization problems based on dual connectivity and backhaul link for 
heterogeneous networks," in 2017 IEEE International Conference on Consumer Electronics - Taiwan (ICCE-TW), Jun. 2017, pp. 229-230. DOI: 10.1109/ICCE-China.2017.7991079.

[32] W. Kim, "Dual connectivity in heterogeneous small cell networks with mmwave backhauls," Mobile Information Systems, vol. 2016, 2016 DOI: $10.1155 / 2016 / 3983467$.

[33] Y. Wu, J. Zheng, K. Guo, L. P. Qian, X. Shen, and Y. Cai, "Joint traffic scheduling and resource allocations for traffic offloading with secrecy provisioning," IEEE Trans. Veh. Technol., vol. 66, no. 9, pp. 8315-8332, Sep. 2017, ISSN: 0018-9545. DOI: 10.1109/TVT. 2017.2689504

[34] A. A. Zaidi, R. Baldemair, H. Tullberg, H. Bjorkegren, L. Sundstrom J. Medbo, C. Kilinc, and I. D. Silva, "Waveform and numerology to support 5G services and requirements," IEEE Commun. Mag., vol. 54 no. 11 , pp. 90-98, Nov. 2016, ISSN: 0163-6804. DOI: 10.1109/MCOM. 2016.1600336CM

[35] 3GPP, "Study on New Radio (NR) acess technology," 3rd Generation Partnership Project (3GPP), TR 38.912, Aug. 2017, v.14.1.0. [Online]. Available: http://www.3gpp.org/DynaReport/38912.htm (visited on 08/03/2017).

[36] S. C. Jha, K. Sivanesan, R. Vannithamby, and A. T. Koc, "Dua connectivity in LTE small cell networks," in 2014 IEEE Globecom Workshops, Dec. 2014, pp. 1205-1210. DOI: 10.1109/GLOCOMW. 2014.7063597

[37] 3GPP, "Study on 3D channel model for LTE," 3rd Generation Partnership Project (3GPP), TR 38.873, Dec. 2016, v.12.3.0. [Online] Available: http://www.3gpp.org/DynaReport/36873.htm (visited on 04/12/2017).

[38] - "Study on channel model for frequencies from 0.5 to $100 \mathrm{GHz}$," 3rd Generation Partnership Project (3GPP), TR 38.901, Sep. 2017 v.14.2.0. [Online]. Available: http://www.3gpp.org/DynaReport/38901. htm (visited on 09/26/2017).

[39] _ - "Evolved universal terrestrial radio access (E-UTRA); physical channels and modulation," 3rd Generation Partnership Project (3GPP), TS 36.211, Mar. 2010. [Online]. Available: http://www.3gpp.org/ftp/ Specs/html-info/36211.htm.

[40] - "Evolved universal terrestrial radio access (E-UTRA); base station (BS) radio transmission and reception," 3rd Generation Partnership Project (3GPP), TS 36.104, Sep. 2012. [Online]. Available: http://www.3gpp.org/ftp/Specs/html-info/36104.htm.

[41] C. Mehlführer, M. Wrulich, J. C. Ikuno, D. Bosanska, and M. Rupp, "Simulating the long term evolution physical layer," in Proc. of the European Signal Processing Conf., Glasgow, Scotland, Aug. 2009 pp. 1471-1478.

[42] 3GPP, "Radio resource control (RRC); protocol specification," 3rd Generation Partnership Project (3GPP), TS 36.331, 2017, v.14.4.0 [Online]. Available: http://www.3gpp.org/DynaReport/36331.htm (visited on 09/25/2017).

[43] E. B. Rodrigues, F. R. M. Lima, T. F. Maciel, and F. R. P. Cavalcanti, "Maximization of user satisfaction in OFDMA systems using utility-based resource allocation," Wireless Commun. and Mob. Computing, vol. 16, no. 4, pp. 376-392, 2014, ISSN: 1530-8677. DOI: $10.1002 / \mathrm{wcm} .2526$

[44] R. P. Antonioli, E. B. Rodrigues, T. F. Maciel, D. A. Sousa and F. R. Cavalcanti, "Adaptive resource allocation framework for user satisfaction maximization in multi-service wireless networks," Telecommun. Syst., pp. 1-17, 2017. DOI: 10.1007/s11235-017-0391-3.

[45] 3GPP, "3GPP specification series: Radio technology beyond LTE," 3rd Generation Partnership Project (3GPP), Tech. Rep. [Online]. Available: http : / / www . 3gpp . org / DynaReport / 38 - series . htm (visited on 04/12/2017)

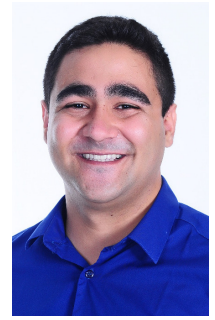

Roberto Pinto Antonioli received his B.Sc. degree in Teleinformatics Engineering (magna cum laude) from the Federal University of Ceará (UFC), Fortaleza, Brazil, in 2016. In 2017, he received his M.Sc. degree in Teleinformatics Engineering also from UFC. He is currently a Ph.D. student and researcher at the Wireless Telecom Research Group (GTEL), UFC, where he works on projects in technical and scientific cooperation with Ericsson Research. In 2014, he was a research assistant with the Data Communications Group at the University of British Columbia (UBC), Canada, where he worked with in-vehicle power line communications. In 2018, he was a visiting researcher at Ericsson in Luleå, Sweden, for 5 months. His research interests include $5 \mathrm{G}$ wireless communication networks with multiple radio access technologies and multi-connectivity, as well as scheduling algorithms for QoS/QoE provisioning.

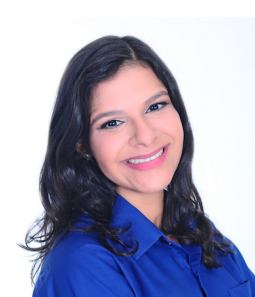

Gabriela Costa Parente Gabriela Costa Parente received the B.Sc. degree in telecommunications engineering from the Federal University of Ceará (UFC), Fortaleza, Brazil, in 2017. From 2016 to 2017, she worked with the Wireless Telecom Research Group (GTEL), UFC, as a research student in a project in cooperation with Ericsson Research. In 2014, she was awarded with a Brafitec scholarship and was an exchange student for 14 months at the École Supárieure d'Électricitá, campus Gif-sur-Yvette, France. Currently, she studies telecommunications engineering at the Institut National des Sciences Appliquées (INSA) de Lyon, France.

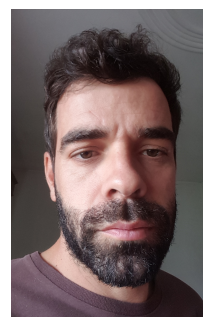

Carlos F. M. e Silva received a five years diploma degree and MSc degree in Electronics and Telecommunications Engineering from the University of Aveiro (UA), Portugal, in 2005 and 2010, respectively. In 2015 he also received a $\mathrm{PhD}$ degree in Teleinformatics Engineering from the Federal University of Ceará (UFC), Brazil. Since 2006, Carlos Silva has been a researcher in several European projects, such as WINNER II (system requirements for beyond 3rd generation wireles networks), FUTON (RRM for wireless and optica networks), and COGEU (cognitive radio systems for efficient use and sharing of TVWS in the European context). Currently, he has a postdoc position at the Wireless Telecom Research Group (GTEL), Brazil, where he manages GTEL's team in the European-Brazilian project FUTEBOL and also works in cooperation projects with Ericsson Research. His main research interests include: spectrum usage optimization, TV White Spaces, Licensed Shared Access, Internet of Things, and Device-to-Device communications; testbed experimentation; and architectural aspects of the future $5 \mathrm{G}$ networks.

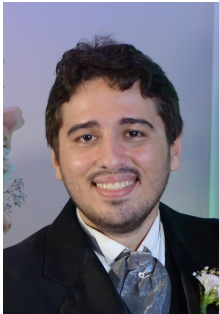

Diego Aguiar Sousa received the B.Sc. degree in Computer Engineering in University of Ceará (UFC), Sobral, Brazil, in 2011. He received M.Sc and Ph.D. degree in Teleinformatics Engineering from the UFC, Fortaleza, Brazil, in 2013 and 2018 respectively. Since 2013, he has been a researcher at the Wireless Telecom Research Group (GTEL), UFC, participating of projects in a technical and scientific cooperation with Ericsson Research. Also, since 2013, he has been a Professor of the Federal Institute of Education, Science, and Technology of Ceará (IFCE), Paracuru, Brazil. His research interests include numerical optimization, 5G networks, coordinated scheduling, radio resource allocation for QoS/QoE provisioning. 


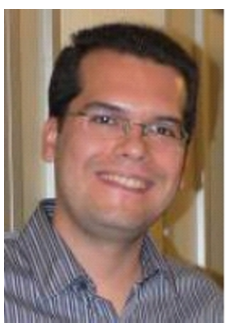

Emanuel Bezerra Rodrigues received his B.Sc. and M.Sc. degrees in Electrical Engineering from the Federal University of Ceará (UFC), Fortaleza, Brazil, in 2001 and 2004, respectively. He also received his Ph.D. degree with honors in Signal Theory and Communications from the Universitat Politècnica de Catalunya (UPC/BarcelonaTech), Barcelona, Spain, in 2011. In 2014, he joined UFC, where he is an Assistant Professor in the Department of Computer Science. He has been working in the Wireless Telecom Research Group (GTEL) since 2001 and has actively participated in several projects in cooperation between GTEL and Ericsson Research. Within this cooperation, he has been in an internship at Ericsson Research at Linköping, Sweden, in 2004. During the last years, he has published several conference papers, journal/magazine articles, and book chapters and has been a reviewer of important international conferences and IEEE journals and magazines. His main research interests are Internet of Things, and radio resource management and QoS control for next-generation mobile and wireless networks.

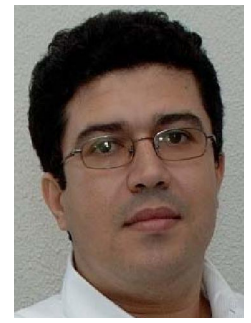

Tarcisio Ferreira Maciel received his B.Sc. and M.Sc. degrees in Electrical Engineering from the Federal University of Ceará (UFC) in 2002 and 2004, respectively, and his Dr.-Ing. degree from the Technische Universität Darmstadt (TUD), Germany, in 2008, also in Electrical Engineering. Since 2001, he has actively participated in several projects in a technical and scientific cooperation between Wireless Telecom Research Group (GTEL), UFC, and Ericsson Research. From 2005 to 2008, he was a research assistant with the Communications Engineering Laboratory, TUD. Since 2008, he has been a member of the Post-Graduation Program in Teleinformatics Enginnering, UFC. In 2009, he was a Professor of computer engineering with UFC-Sobral and since 2010, he has been a Professor with the Center of Technology, UFC. His research interests include radio resource management, numerical optimization, and multiuser/multiantenna communications.

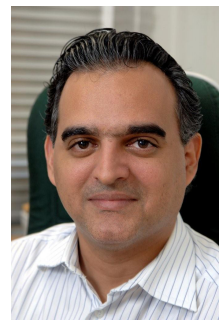

Francisco Rodrigo Porto Cavalcanti received the B.Sc. and M.Sc. degrees in Electrical Engineering from Federal University of Ceará (UFC), Fortaleza, Brazil, in 1994 and 1996, respectively, and the D.Sc. degree in Electrical Engineering from the State University of Campinas, São Paulo, Brazil, in 1999. Upon graduation, he joined the UFC, where he is currently an Associate Professor and holds the Wireless Communications Chair with the Department of Teleinformatics Engineering. In 2000, he founded and, since then has directed the Wireless

Telecom Research Group (GTEL), which is a research laboratory based on Fortaleza, which focuses on the advancement of wireless telecommunications technologies. At GTEL, he manages a program of research projects in wireless communications sponsored by the Ericsson Innovation Center in Brazil and Ericsson Research in Sweden. Prof. Cavalcanti has produced a varied body of work including two edited books, conference and journal papers, international patents and computer software dealing with subjects such as radio resource allocation, cross-layer algorithms, quality of service provisioning, radio transceiver architectures, signal processing and project management. Prof. Cavalcanti is a distinguished researcher of the Brazilian Scientific and Technological Development Council for his technology development and innovation record. He also holds a Leadership and Management professional certificate from the Massachusetts Institute of Technology, Cambridge. 